\title{
Integral hospital response to emergencies and disasters in a private hospital in Mexico
}

\author{
Elisa Naeme Saleme-Cruz ${ }^{1}$, Luz Angélica de la Sierra - de la Vega ${ }^{2 *}$ and José Luis Kramis-Cerezo ${ }^{3}$ \\ ${ }^{1}$ The American British Cowdray, Ciudad de México, CDMX, Mexico \\ ${ }^{2}$ Environmental Health Department, Center for Population Health Research. National Institute of Public Health Cuernavaca, Mexico \\ ${ }^{3}$ International Comitee Red Cross, Mexico
}

\begin{abstract}
In this manuscript, we show that the design and implementation of a hospital response protocol for emergencies and disasters is essential to offer an effective health response attached to international programs aimed at risk reduction, which allow the identification of personnel and the actions to be taken during a critical event. Our purpose was to evaluate their previous knowledge and actions in similar situations, as well as to detect the strengths and areas of opportunity of each organization. This brief article describes the perceptions of the staff of a private hospital in Mexico City about their individual and institutional actions in emergencies and disasters, collected through questionnaires and semi-structured interviews.
\end{abstract}

\section{Introduction}

Mexico has been the subject of many social and natural events that have tested its capacity for health response, especially in matters of infrastructure and Resources [1].

The second and third level hospital units are an axis of central response and reference in this type of events, so guaranteeing their integral response, organized and with a decisive vision, promotes a strategy of prevention and mitigation of risk in emergencies and disasters [2].

The frequency of climate-related disasters is increasing, since in the last twenty years an annual average of 30,000 lives has been claimed and caused more than 4,000 million injured. According to the UN, $90 \%$ of natural disasters are related to the climate, while the remaining $10 \%$ are of geophysical origin (earthquakes, volcanic eruptions and landslides). In the first group, the deadliest and most serious effects are floods and droughts, causing $80 \%$ of the victims and which are predominantly poor countries [3].

Mexico and in particular Mexico City present special challenges, such as overpopulation and poor access to sanitation services. The most destructive event with greater repercussions, has been the earthquake that occurred in 1985, with approximately 6000 deaths, generating an approximate cost of $\$ 4.1$ billion, which showed the inability of the system to face events of similar magnitude [4]. This situation was faced based on the national and international solidarity social response. There were no trained personnel in the areas of critical response for the reception of the injured, rescue, much less with organizational principles to provide an effective health response. Recovering the evidence and statistics of the repercussions of these events, the Pan American Health Organization (PAHO) promoted a resolution (CD 45.R8) demanding that member states adopt a policy on risk reduction the initiative of "Safe Hospital against disasters "in such a way as to ensure their capacity to respond and function in critical situations. This was adopted by 168 countries, including Mexico, in 2005 in the framework of the "World Conference on Disaster Reduction" in Kobe
Hyogo, Japan [5]. This highlights the need for priority attention to multiple victims and the support and organization of medical services. With central axes the protection of life, infrastructure and function [6].

The proper functioning of health services depends on several important aspects, including functionality and integrity in the provision of vital services and the adequate supply of supplies [5,7].

In these situations, some of the scenarios to be analyzed are the massive flow of patients with injuries that may or may not be of traumatic origin, patients with chronic conditions that derive from this type of sudden situation require in-hospital management and finally the increase in intracommunicable diseases and outpatient $[8,9]$.

This increase in the demand for services requires an organized reaction of administrative, technical and operational processes, before, during and after the response to the event.

Therefore, regardless of the origin of the Emergency or Disaster, it is essential to have a unique plan of action, which guides and guides the areas that lead the response to promote an effective limitation of damage, not only to the health structure, but also in social costs. It is extremely important to have a plan that allows consecutive steps to be taken and that ensures timely and safe patient care [9].

This requires that all people involved in the design, direction, performance and control of the plans are motivated to develop a high level of awareness and commitment, resulting in a more active participation in the search for integral solutions $[10,11]$.

${ }^{*}$ Correspondence to: Luz Angélica de la Sierra de la Vega, Professor and researcher, Center for Population Health Research. National Institute of Public Health Cuernavaca, Mexico, Tel: (52) 7773293000 Ext. 3301; E-mail: luz. delasierra@insp.mx

Received: September 24, 2019; Accepted: November 12, 2019; Published: November 21, 2019 
The American British Cowdray (ABC) Medical Center (Santa Fe Campus) is a third level private nonprofit assistance institution located in the west of Mexico City. It has a staff of 1400 doctors of 40 specialties and a care capacity of 154 beds.

In the present study, all the variables that influence the hospital response that should be considered in the creation of a comprehensive hospital response protocol in cases of emergencies and disasters were identified, to reduce the consequences and impact of events that may occur in the $\mathrm{ABC}$ Center Medical, reducing costs and offering an essential solution for its continuity and applicability.

\section{Materials and methods}

We performa a cross-sectional and descriptive study with a qualitative and quantitative methodology, which allowed to generate the bases for the development of a hospital protocol for emergency and disaster response in the $\mathrm{ABC}$ Medical Center, Santa Fe Campus.

A questionnaire was applied to 30 workers who participate in the response to emergency and disaster events chosen by a convenience sampling. The questionnaire was divided into 4 sections: in the first, general information was obtained from the participants; the second constituted the "before the disaster" phase: the risks and threats that they recognize for the hospital; emergency or disaster training. The second section or "phase during" in terms of actions and activation to be performed. Finally, a section for the Emergency team was included because of the qualities and knowledge that they should know about the care protocols and finally a section for the members of the Crisis Committee, key operators in these types of situations.

To complement the information, 10 semi-structured interviews were conducted with personnel participating in the hospital response to emergencies and disasters. Sampling was carried out for convenience considering operational and command workers of the critical areas involved. The interview guide included sections: attention experience; success in response; improvement points; and importance of the existence of a hospital response protocol. The quantitative information analysis was carried out through the stata 14 program, through which descriptive statistics were obtained. Qualitative information was analyzed through the Atlas ti software, making a matrix defining analytical categories according to the sections of the interview guide.

\section{Results}

\section{Quantitative analysis. Stage Before the event}

All participants considered it's very important to have a plan that allows for consecutive steps and that ensures timely and safe patient care, considering it an ethical and social obligation. The average age was 30.7 years; $56.6 \%$ participants belong to the female sex. Regarding schooling, we found that they mostly have some academic degree, higher than basic education, $46.6 \%$ with a degree mostly from the health or administrative area and $27 \%$ with postgraduate education.

It was observed that the female sex has a predominance of professional training in relation to the male participants, which is directly related to their employment position in the Institution. $45 \%$ of the respondents belong to a headquarters / or are in charge of the Service of origin. Of the total respondents, only $45 \%$ are in the Crisis Committee. $85 \%$ of participants consider that there are risks and threats that can have a direct effect on the hospital. The total participants consider it as a social and ethical responsibility to be prepared to respond to an emergency or disaster situation. As for the external threats that respondents identify can affect the hospital, they predominantly point to earthquakes and fires / explosions as the main ones. It was interesting to analyze their knowledge regarding the training received, $90 \%$ reported having it being an access requirement to work especially in hospital areas. Regarding $83.3 \%$ reported having been trained in person (course) or at the time of a drill.The options that the hospital offers for such training vary: $60 \%$ report having been trained through an in-person course, an online course, scheduled drills and instructional responsibility by the immediate bosses.

Only $10 \%$ of them report having been instructed by all possible methods, while $60 \%$ indicate that they received instruction by at least 2 methods (classroom course and simulation). Regarding the knowledge about the Crisis Plan of the ABC Medical Center (document that sets out some chains of action in case of internal emergency, the organizational chart of the Crisis Committee, the meeting points), $80 \%$ of the participants know the same and in almost similar figures, (85\%) know where they can locate it for consultation.

\section{Stage During an Emergency or Disaster Situation}

When questioning participants about their knowledge about warning / alert and activation systems in an Emergency or Disaster situation at $\mathrm{ABC}$ Medical Center, only 3.3\% said they did not know it.Regarding the services that pay attention during this type of situation, 93.3\% stated that they identified those services that should do it and that their service was part of the necessary response. Only $13 \%$ of the participants did not identify their service as a response provider, although within the actions all the services of the participants are required in it.

It was questioned whether at the time of answering an algorithm in their service that was used as an action guide, $40 \%$ of the participants did not identify any type of action algorithm in their service.

Finally, the question we asked about who should be in charge of directing and organizing the response in their service of origin, having as an answer that the Head of the Service or they themselves would have that function.

Emergency Service. Questioned about the knowledge of codes for activation of the warning or assistance of other hospital areas in a critical situation, everyone answered positively.

However, at the time of questioning during this type of event, who should carry out the activation, $56.6 \%$ indicated to the Chief of the Emergency Department as the person in charge of the activation, the rest stated that it was a partner or themselves who should activate it. $100 \%$ of participants indicated knowing the activation process and the expected response (assistance and mobilization) by other services and their role in this response to Emergencies or Disasters.

\section{Crisis Committee}

It was analyzed if there was knowledge among the members of the Crisis Committee, finding that $83.3 \%$ of the people know the rest of the members. When asked if everyone knows their function, $100 \%$ of them, refer to doing so.At the time of the activation of the code, the members of the Crisis Committee were questioned, how was the form or mechanism by which they maintained communication with the operational personnel that were responding in the Emergency and Disaster situation, predominated such that communication to the personal telephone is the most used way to do it.

It is important to mention that in addition to the members of the Committee to which the questionnaire was made, at least 8 people believed they were from that committee, without actually belonging to it. 


\section{Qualitative analysis. Experience in attention}

Participants consider here has been adequate coordination at the time of the response to the care of multiple victims. For many of them, this situation need "precise, timely, skillful" attention. The majority expressed the impact that their work has on the population.

\section{Success in the answer}

All participants consider that the response so far has been successful, but there are aspects that could be improved. They refer to the training support they have received to face similar situations. However, at least 4 participants clarify that there is no written protocol to adhere to, the result has simply been secondary to the empirical organization.

\section{Improvement points}

The participants consider that the organization of the staff is elementary, that the existence of a protocol would help much independent of the type of patient (sex, age, health condition, etc.).

Emphasis is placed on the continuous training of personnel, and familiarize themselves with the Emergency Department (in relation to what these are, the entrance door and those leading the response).

Some interesting ideas are reflected on the need for communication between the administrative and operational area.

\section{Importance of the existence of a hospital response protocol}

All participants were very blunt and concise in the response, they are clear about the need for a protocol that allows them to act better and in a more organized way. Participants believe that having a protocol would guarantee better patient care and would provide an "efficient, timely and quality" service. Some refer would feel more confident and able to provide the answer and even "generate direction to work.

\section{Discussion and conclusions}

The ABC Medical Center Santa Fe Campus has experience in the mass care of victims with good results, who have managed to improve the prognosis of patients' lives. In emergency or disaster situations, one of the main elements that generates concern is the consequences on the population. The physical and emotional injuries derived from this type of situations and the experience of the population about them, generate a collective conscience in search of safer situations and try to organize an adequate response, not only at the level of the rescue systems of victims, but also of health organizations [12].

These critical situations will continue to be a social and health issue that requires not only the commitment of the community but also of the people who work in the hospital centers.

In any community, the hospital is an axis of reference and has a greater political and social role, unlike even other essential establishments. Hospitals also represent a social investment, from which it should be benefited. The expectation of the population is to receive medical attention and at the same time feel safe in specific situations in which they are vulnerable [13].

Having established protocols for the care of victims arising from an emergency or disaster situation is an ethical responsibility that all health institutions should comply with [14].

In developing countries such as Mexico, with poor access and health coverage, with a supersaturation of hospitals, it is difficult to think about creating protocols or plans for the care of multiple victims. Sometimes it is only necessary to reorganize the resources and priorities and give room to trained personnel to implement these types of protocols [15].

Without a doubt, the protection of life is the elementary principle and the foundation of the medical task, however, it is complexity in critical situations and with multiple victims, its importance extends to protecting health public before, during and after emergencies or disasters $[13,16]$.

Health workers become a primary focus in crisis situations and at this time, through this experience, there is a political and social will worldwide, to improve decisions and actions in disaster risk reduction, particularly in the system of Health $[13,10]$.

In Mexico, hospitals are required to achieve the objectives of Safe Hospital in regards to their safety and responsiveness. Personnel should definitely be trained and protected since when hospitals are destroyed or damaged, it is more difficult to save lives [17].

The initiative of Safe Hospitals, demonstrates that it is possible to evaluate in an integral and efficient way the capacities of a health Institution and that large amounts of economic resources must not be spent for its implementation. The responsibility of ensuring that the principles of safe hospitals are reproduced and achieved must be shared by the health authorities and all those who work in the health system, regardless of whether it is within a public or private environment. A mistake often made is to devalue private institutions; however, many management models that can be reproduced in a public health environment can be learned from them $[18,10]$.

\section{Ethical considerations}

This was a Minimum Risk Study, based on the following considerations:

Internal Regulations of the Research Ethics Committee of the National Institute of Public Health. Regulation of the General Law of Health in Research Matters. The information collected in the present study was treated in a strictly confidential manner, uniquely and exclusively was used by the study researchers without availability for another purpose. The results of the study were used for academic / scientific purposes There are no conflicts of interest on the part of the authors.

\section{Conflict of interest}

The authors declare no conflict of interest.

\section{Author contribution}

Elisa Saleme Naeme-Cruz, Luz Angélica de la Sierra-de la Vega and José Luis Kramis-Cerezo conceived of the presented idea. Elisa Saleme Naeme-Cruz carried-out data collected and analysis of interviews and questionaries. Luz Angélica de la Sierra-de la Vega and José Luis Kramis-Cerezo supervised the findings of this work. All authors provided critical feedback and helped shape the research, analysis and manuscript.

\section{References}

1. CENAPRED, National Civil Protection System (2016) Socioeconomic impact of the Main Disasters ocurred in Mexico in the year 2015. Ministry of the Interior, Mexico City.

2. National Civil Protecion System (2017) Ministry of the interior Practical Guide of the Safe Hospital Program. Mexico System, Mexico.

3. PAHO/WHO/World Bank (2013) Steps for the preparation of the Hospital plan for Emergency and Disaster Response. Guide for the preparation of the Hospital Plan for Emergency and Disaster Response. Santo Domingo pp: 1-18. 
4. Secretaría de Gobernación/Secretaría de Salud (2014) Strategic Hospital Insurance Plan.

5. http://iris.paho.org/xmlui/handle/123456789/49736

6. http://www.planeamientohospitalario.info/

7. PAHO (2013) Risk Reduction and Disaster Response. Strategic Plan 2013-2018, Washington, D.C.

8. Ministry of the Interior (2017) Safe Hospital Program, Inter insitutional Evaluation 2014. Ministry of the Interior, Mexico.

9. Gutiérrez, Gregorio y Parra, Liz (2013) Generalities in Safe Hospitals. PAHO Regional Office for the Americas. pp: 13-38.

10. PAHO (2013) Systematization of the experience of Safe Hospital in the Dominican Republic. Santo Domingo 155: 1.

11. Information and communication management in the Emergencies and Disaster (2009) CCPSD newsletter p. 15.

12. Del Busto-Prado, Francisco, Arcos-González, Pedro Ignacio y Castro-Delgado, Rafael (2019) Disasters and Public Health: An Aproach from the Theoretical Framwork of Epidemiology. Ministry of Health, Social Services and Equality, Madrid, Spain.
13. Nekoie-Moghadam M, Kurland L, Moosazadeh M, Ingrassia PL, Della Corte F, et al (2016) Tools and Checklists Used for the Evaluation of Hospital Disaster Preparedness: A Systematic Review. Disaster Med Public Health Prep 10: 781-788.

14. Morales, Nelson. 1, Lima, Peru (2001) Some considerations for the Organization of Health Services for Emergencies and Disasters. Universidad Nacional Mayor de San Marcos, Anales de la Facultad de Medicina.

15. Sánchez-Torres, Diana Alejandra (2017) Accesibility to health services: theoretical debate on determinants and implication in public health policy. Rev Med Inst Mex Seguro Soc 55: 82-89.

16. James, JJ, y otros (2010) Disaster medicine and public health preparedness: a discipline for health professionals 4: 102-107.

17. Sheikhbardsiri H, Raeisi AR, Nekoei-Moghadam M, Rezaei F (2017) Surge Capacity of Hospitals in Emergencies and Disasters With a Preparedness Approach: A Systematic Review. Disaster Med Public Health Prep 11: 612-620. [Crossref]

18. PAHO/WHO (2019) Health in the Americas summary: regional panorama and country profiles.

Copyright: (C2019 Saleme-Cruz EN. This is an open-access article distributed under the terms of the Creative Commons Attribution License, which permits unrestricted use, distribution, and reproduction in any medium, provided the original author and source are credited. 